\title{
The Hemarthrosis-Simulating Knee Model: A Useful Tool for Individualized Education in Patients with Hemophilia (GEFACET Study)
}

This article was published in the following Dove Press journal: Journal of Blood Medicine

\author{
Sophie le Doré \\ Nathalie Grinda ${ }^{2}$ \\ Emmanuelle Ferré \\ Valerie Roussel-Robert ${ }^{3}$ \\ Birgit Frotscher ${ }^{4}$ \\ Pierre Chamouni ${ }^{5}$ \\ Sandrine Meunier ${ }^{6}{ }^{6}$ \\ Sophie Bayart ${ }^{7}$ \\ Edita Dolimier ${ }^{8}$ \\ Francoise Truong-Berthoz ${ }^{9}$ \\ Emmanuelle de Raucourt' \\ 'Haemophilia Treatment Centre, Hôpital \\ Mignot, Versailles, France; ${ }^{2}$ Regional \\ Hemophilia Centre, Hôpital de Bicêtre \\ (AP-HP), Kremlin-Bicêtre, France; \\ ${ }^{3}$ Regional Hemophilia Treatment, Hôpital \\ Cochin (AP-HP) Centre, Paris, France; \\ ${ }^{4}$ Haemophilia Treatment Centre, Hôpital \\ de Brabois, Vandoeuvre-Les-Nancy, \\ France; ${ }^{5}$ Regional Hemophilia Treatment \\ Centre, Centre Hospitalier Universitaire \\ de Rouen, Rouen, France; ${ }^{6}$ Hospices \\ Civils de Lyon - Unité d'Hémostase \\ Clinique, Hôpital Cardiologique Louis \\ Pradel, Bron, France; ${ }^{7}$ Haemophilia \\ Treatment Centre, Hôpital Pontchaillou, \\ Centre Hospitalier Universitaire de \\ Rennes, Rennes, France; ${ }^{8}$ Baxalta France \\ SAS, a Takeda Company, Paris, France; \\ ${ }^{9}$ Baxalta $\mathrm{GmbH}$, a Takeda Company, \\ Zurich, Switzerland
}

Correspondence: Emmanuelle de Raucourt

Service d'Hématologie Biologique, Clinique des Anticoagulants (CAC), Unité Inserm U II48, Hôpital Beaujon, Clichy, France

Tel +33 0l 4087554 I

Fax +33 ol 40875683

Email emmanuelle.de-raucourt@aphp.fr
Background: Hemophilic arthropathy is a major complication in patients with severe hemophilia. A plastic knee model has been developed for the therapeutic education of patients to promote improved care management and self-treatment skills. The objective of this study was to evaluate the impact of this hemarthrosis-simulating artificial knee (HSAK) on patients' knowledge of their disease and its treatment.

Methods: In this observational study, the impact of HSAK was assessed during individualized education in patients with severe/moderately severe hemophilia A or B at seven hemophilia treatment centers in France. Participants provided written informed consent and completed questionnaires to assess knowledge of their disease (score range: 0-7) and knowledge of their treatment (score range: 0-4). Questionnaires were completed before, immediately after and 6 months after HSAK use. The scores obtained before and after the use of the HSAK were compared.

Results: The participants comprised 32 children, 29 teenagers, and 31 adults. The mean (SD) disease knowledge score increased significantly in all age groups of patients from 4.5 (2.0) to $5.9(1.5 ; \mathrm{p}<0.001)$ immediately after the training and remained unchanged at 6 months. Mean (SD) treatment knowledge scores were unchanged, but Wilcoxon signed rank testing showed a significant increase after the training course that was maintained at 6 months in children and teenagers.

Conclusion: These findings suggest that an individualized training course can enhance the understanding of hemophilia in patients of all ages, especially in children and teenagers, and that the HSAK may assist in improving patients' management of their disease.

Keywords: hemarthrosis, hemophilia, knee joint, patient education as topic, therapeutic education

\section{Introduction}

One of the major complications of hemophilia, especially in patients with severe or moderate disease, is recurrent joint bleeding leading to musculoskeletal complications. $^{1,2}$ If intra-articular bleeding is poorly controlled it can lead to the development of chronic synovitis and multi-articular arthropathy. ${ }^{3,4}$ Diagnosis and treatment of these bleeding episodes must be delivered as early as possible and intensive treatment should be performed until the resolution of symptoms. ${ }^{5-10}$ Early prophylactic treatment could avoid the development of hemophilic arthropathy. ${ }^{11-14}$ Low compliance with prophylaxis may limit the effectiveness of treatment. ${ }^{15}$ Education of patients with hemophilia and their families helps reduce mortality 

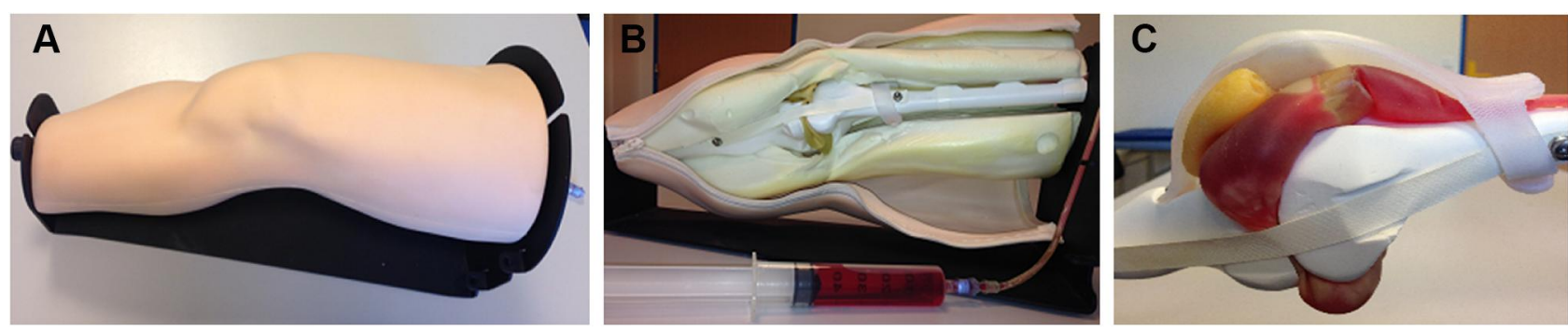

Figure I HSAK overview: the outer view (A), inner view (B), and fillable artificial joint capsule (C) of the hemarthrosis-simulating artificial knee.

and morbidity and may boost their quality of life. ${ }^{16,17}$ This education should include information about the nature of the disease and its complications as well as the therapeutic products available and home treatment strategies, including self-administered intravenous infusions and selfmanagement. Lifelong supervision of patients is essential to reinforce self-treatment, self-management, adherence and coping strategies. ${ }^{18,19}$

The hemophilia treatment center (HTC) at the Hôpital de Versailles, France, has developed a novel hemarthrosissimulating artificial knee model (HSAK) (Figure 1) for use as a therapeutic education (TE) tool. The knee model was initially tested on a small group of patients at the Versailles HTC. After reports of improvements in the management of care and patients' self-treatment skills, an observational study was designed to evaluate the impact of this tool on patients' knowledge. This study was called GEFACET, the French acronym for "Artificial Knee and Therapeutic Education."

\section{Materials and Methods}

The study was conducted between May 2015 and February 2017 at seven hemophilia treatment centers in France: (1) Hôpital de Versailles, Versailles, (2) Regional Hemophilia Centre, Hôpital de Bicêtre (AP-HP), KremlinBicêtre, (3) Regional Hemophilia Treatment Centre, Hôpital Cochin (AP-HP), Paris, (4) Haemophilia Treatment Centre, Hôpital de Brabois, Vandoeuvre-LesNancy, (5) Regional Hemophilia Treatment Centre, Centre Hospitalier Universitaire de Rouen, Rouen, (6) Hospices Civils de Lyon - Unité d'Hémostase Clinique, Hôpital Cardiologique Louis Pradel, Bron, and (7) Haemophilia Treatment Centre, Hôpital Pontchaillou, Centre Hospitalier Universitaire de Rennes, Rennes. Investigators (physicians, physiotherapists, psychologists, nurses) invited all patients with severe or moderately severe hemophilia A or B who were receiving therapeutic education with the HSAK to participate in the study. All participants (patients or their legal representatives) provided written informed consent to take part in the study, which was undertaken in accordance with the Declaration of Helsinki. This project on educational intervention with the HSAK was strictly observational. Only questionnaires were used during the study and no clinical or biological data were recorded. The CCTIRS (French Advisory Board on Data Processing and Methodology) granted a favorable opinion. Authorization from CNIL (French Data Privacy Regulatory Agency) was obtained on 16th February 2015: authorization number 914,637.

The knee model was initially developed for healthcare staff training to visualize synovial fluid aspiration. It was then adapted to provide a visual effect of hemarthrosis on the anatomy of the knee joint and the impact of treatment (Figure 1). The model includes a pouch that can be filled with red liquid representing blood, thereby simulating hemarthrosis. When the red liquid is injected, the external aspect of the knee swells and the accumulation of blood in the joint is visible. A second pouch filled with a clear liquid is then used, showing the effect of treatment with coagulation factor concentrate on hemarthrosis. When this clear liquid is injected, the red liquid is progressively removed and the knee recovers a normal aspect. The presentation was standardized for all sites and is described in the Supplementary Section.

Participants were asked to complete three questionnaires specifically developed for the study. Two questionnaires were completed on the day of enrollment (D0). The first collected biometric data (age and sex). The second assessed knowledge of hemophilia and management of hemarthrosis; it was completed before the use of the HSAK. Immediately after completing the training course with the HSAK, patients or relatives were asked to complete the second questionnaire with an additional question on the HSAK. Six $( \pm 1)$ months later (M6 visit), participants were invited to complete a third questionnaire, which was identical to that used just after the education 
session with HSAK at enrollment but included a new question relating to possible treatment stoppage since inclusion. The M6 questionnaire was completed whenever possible at the HTC or at home.

It was left to the discretion of the investigator or patient's legal representative to determine whether minors were able to complete the questionnaires alone. However, all three questionnaires had to be completed by the same person during the study. Patient responses were coded by investigators taking into account age differences.

The primary endpoint of the survey was the score that assessed patient knowledge of the disease. Unanswered questions or incorrect answers scored 0 ; correct answers scored 1. Disease knowledge was calculated on the basis of four questions, with a possible total mark of 7 points. This score was calculated at D0 pre-TE, then at D0 postTE, and finally at M6.

Another score (graded from 0 to 4 ) was calculated for the secondary endpoint, which assessed patients' knowledge of their treatment. This covered patient appreciation of HSAK use as well as patient understanding of the disease and treatment of bleeding episodes. Information on treatment stoppage was provided at M6. Questionnaires and score assessment are detailed in the Supplementary Section.

To ensure the quality of results, data were double entered and data management consistency tests were conducted on the clinical database. Before analysis, a data entry quality control was performed on a representative number of case report forms. Statistical analyses were performed using SASR software (Version 9.4, SAS Institute, North Carolina State University, United States). Quantitative data were described by their numbers, mean, standard deviation (SD), median and extreme values. Qualitative data were described by their numbers and percentage. 95\% bilateral confidence intervals (CI) were provided when deemed relevant. The significance threshold (Type I error) was set to $\alpha=5 \%$. The pre-education and post-education scores and sub-scores as well as scores and sub-scores found at D0 and M6 were compared using appropriate statistical tests: Wilcoxon signed rank test (for questions with at least 3 answers) or MacNemar test (for binary questions).

\section{Results}

Thirty-two children ( $<12$ years of age), 29 teenagers (12-18 years), and 31 adults ( $>18$ years) met the enrollment criteria and were eligible for the study. All 92 patients were male; median age was 14 years, ranging from 6 to 68 years. Most patients $(81.5 \%)$ had severe hemophilia A. Eight dropouts were observed, either at the patient's request $(n=6)$ or lost to follow-up ( $\mathrm{n}=2$ ). The M6 visit was therefore completed by $91.3 \%$ of enrolled patients.

At baseline, the median (mean $\pm \mathrm{SD}$ ) score for disease knowledge in all patients was 5 (4.5 \pm 2.0$)$. This significantly increased after the TE session to $7(5.9 \pm 1.5)$ $(\mathrm{p}<0.001)$, and remained unchanged at M6: $7(5.9 \pm 1.4)$ (Table 1). The change in disease knowledge score was

Table I Scores of Knowledge of Disease and Its Treatment Before and After HSAK Use

\begin{tabular}{|c|c|c|c|c|c|c|}
\hline \multirow[t]{2}{*}{ Age Group } & \multicolumn{3}{|c|}{ Score of Knowledge of Disease } & \multicolumn{3}{|c|}{ Score of Knowledge of Treatment } \\
\hline & D0 Before TE & DO After TE & M6 & D0 Before TE & DO After TE & M6 \\
\hline \multicolumn{7}{|l|}{ Children } \\
\hline & 32 & 32 & 31 & 32 & 32 & 31 \\
\hline Mean \pm SD & $3.4 \pm 2.1$ & $5.4 \pm 1.8^{\mathrm{a} *}$ & $5.7 \pm 1.4^{b *}$ & $2.2 \pm 0.8$ & $3.0 \pm 0.7^{\mathrm{a} *}$ & $3.0 \pm 0.8^{b *}$ \\
\hline \multicolumn{7}{|l|}{ Teenagers } \\
\hline $\mathrm{N}$ & 29 & 29 & 26 & 29 & 29 & 26 \\
\hline Mean \pm SD & $4.6 \pm 1.7$ & $6.3 \pm 1.3^{\mathrm{a} *}$ & $5.9 \pm 1.5^{b} *$ & $2.7 \pm 0.6$ & $3.2 \pm 0.6^{\mathrm{a} *}$ & $3 \pm 0.6$ \\
\hline \multicolumn{7}{|l|}{ Adults } \\
\hline $\mathrm{N}$ & 31 & 31 & 27 & 31 & 31 & 27 \\
\hline Mean \pm SD & $5.5 \pm 1.7$ & $6.1 \pm 1.2 \mathrm{a} *$ & $6 \pm 1.3$ & $2.8 \pm 0.5$ & $2.9 \pm 0.5$ & $3 \pm 0.5$ \\
\hline \multicolumn{7}{|l|}{ All } \\
\hline$N$ & 92 & 92 & 84 & 92 & 92 & 84 \\
\hline Mean \pm SD & $4.5 \pm 2$ & $5.9 \pm 1.5 \mathrm{a} *$ & $5.9 \pm 1.4^{b *}$ & $2.6 \pm 0.7$ & $3 \pm 0.6^{a *}$ & $3 \pm 0.7^{b *}$ \\
\hline
\end{tabular}

Notes: Changes in mean disease- and treatment-related knowledge scores ${ }^{a}$ between D0 pre-TE and D0 post-TE and ${ }^{\mathrm{b}}$ between D0 pre-TE and the M6 visit. ${ }^{*} \mathrm{p}<0.00 \mathrm{I}$ as determined by Wilcoxon signed rank test (nonparametric intraindividual data comparison).

Abbreviations: D0, day of enrollment; M6, $6( \pm I)$ months after enrollment; TE, therapeutic education. 
significant $(\mathrm{p}<0.001)$ in all groups of patients: children, teenagers and adults at D0 after TE (Table 1). For all patients, the disease awareness score had significantly improved at the M6 visit compared with the D0 pre-TE score $(\mathrm{p}<0.001)$, with a median increase (mean \pm SD) of 1 (1.3 \pm 2.0$)$. Sub-group analysis revealed that the change in disease knowledge score at the M6 visit was significant only in children and teenagers (Table 1).

At baseline, the median (mean $\pm \mathrm{SD}$ ) score for treatment knowledge was 3 (2.6 \pm 0.7$)$. This remained at 3 (3.0 $\pm 0.6)$ after the TE session and at M6 (3.0 \pm 0.7$)$ (Table 1). A Wilcoxon signed rank test revealed that the score had significantly increased after TE $(\mathrm{p}<0.001)$, and that this increase was maintained at M6 ( $<<0.001)$. Sub-group analysis revealed that this increase was significant only in children and teenagers at D0 after TE and in children at M6 (Table 1).

In addition, 61 (66.3\%) and 48 (52.2\%) patients commented on D0 and at the M6 visit, respectively, that the HSAK helped them "a lot" to understand hemarthrosis after receiving TE. Similarly, most patients (64.1\%) considered that the HSAK helped them "a lot" to understand explanations provided by healthcare providers on D0 after TE; this figure was $47 \%$ at the M6 visit. However, patients expressed mixed opinions about whether their disease knowledge or understanding of how hemarthrosis is treated had been improved through training.

Of the 84 patients who completed the M6 visit, only one patient $(1.2 \%)$ stopped prophylaxis in the 6 months prior to the M6 visit.

\section{Discussion}

This study aimed to assess the impact of using the HSAK on disease- and treatment-related knowledge in male patients, most of whom had severe hemophilia A. The HSAK used in this TE program for children, adolescents, and adults improved the knowledge of the disease among these patients and their caregivers. Overall, this improvement was maintained 6 months after TE compared with the baseline (ie, before using the HSAK), although this improvement was significant only in children and teenagers at M6 compared with D0 before the educational intervention. Interestingly, age group analysis also showed that improvement of the knowledge of treatment at D0 after TE was statistically significant for children and teenagers but not for adults. These findings suggest that an individualized program provided at any age can enhance the understanding of hemophilia, especially in children and teenagers. In addition, our results suggest that the HSAK, as a novel educational device, could enable patients to be more effective in looking after themselves. ${ }^{20}$

This study also showed that most patients had a high level of knowledge regarding their treatment and were aware of the value of prophylaxis, even when joint pain and swelling had subsided. Educating patients and their caregivers about the disease and treatments to prevent bleeding damage is essential for ensuring treatment adherence: caregiver acceptance, infrequent bleeds, venous access, and treatment compliance have been reported to be possible obstacles to adherence in pediatric patients with hemophilia. ${ }^{21-24}$ Alternatively, educational efforts could specifically focus on patients entering the transitional period from childhood to adolescence, because adolescents appear to be more reluctant to use treatment than younger patients. ${ }^{25}$ Interestingly in the present study, improvements of disease and treatment knowledge were generally greater in young patients than in adults.

This study is limited owing to several biases inherent in the way the data were collected: patient questionnaires can be interpreted differently and are sometimes completed indifferently by patients (because they have to reply three times to the same questions). However, few studies have reported evaluation of educational materials and educational intervention is usually evaluated on patient questionnaires. ${ }^{20,26,27}$ The interval between TE and the M6 visit $( \pm 1)$ also differed between patients, and might have affected how their knowledge levels were assessed 6 months after training. In addition, there was no standard method for dealing with dropouts in this study. Some patients who did not attend their M6 visit when the clinical research associate (CRA) monitored the study sites were excluded from the study, whereas data were collected from some patients who returned their questionnaire more than 7 months after the baseline visit but prior to the CRA monitoring visit. Furthermore, no comparison was made between TE without the HSAK and TE with the HSAK, although this could be done in a further study. Finally, the small sample size included in each sub-group was a limitation although this size may be relevant given that hemophilia is a rare disease.

Despite these limitations it is noteworthy that about two-thirds of patients said they appreciated the use of this device; the HSAK was especially appreciated by younger patients because its visual nature allowed them to understand more easily. These findings should be 
confirmed by further studies, and it may be possible to develop similar models for other joints.

\section{Conclusions}

The results of the GEFACET study, based on the completion of questionnaires after training performed by healthcare providers using the HSAK, showed a significant improvement in patient knowledge concerning hemophilia and its treatment, especially in children and teenagers. They suggest that the HSAK could be used as a support tool for TE, and might be valuable for helping patients with hemophilia to improve their disease management in an everyday setting. These findings should be confirmed by further studies.

\section{Abbreviations}

CRA, clinical research associate; D0, day of enrollment visit; GEFACET, artificial knee and therapeutic education [in French]; HSAK, hemarthrosis-simulating artificial knee; HTC, hemophilia treatment center; M6, 6-month visit; TE, therapeutic education.

\section{Acknowledgments}

We thank all investigators and healthcare providers involved in the study. We also wish to acknowledge Axonal Biostatem France SAS for its support in study management, monitoring, data management, statistical analysis, and medical writing. Additional editorial support for this manuscript was provided by Jackie van Bueren, BSc, employee of Excel Medical Affairs (Fairfield, CT, USA), and was funded by Baxalta US Inc, a Takeda company, Lexington, MA, USA. All authors were involved in writing the manuscript and have approved the final article. Preliminary results of this paper were presented as a poster to the Annual Congress of the European Association for Haemophilia and Allied Disorders 2017, 1-3 February 2017, Paris, France; https://onlinelibrary. wiley.com/doi/full/10.1111/hae. 13150

The abstract of this paper was presented as a poster to the Annual Congress of the European Association for Haemophilia and Allied Disorders 2019, 6-8 February 2019, Prague, Czech Republic; https://onli nelibrary.wiley.com/doi/full/10.1111/hae.13666.

\section{Funding}

The GEFACET study was funded by Baxalta France SAS, a Takeda Company, Paris, France. The scientific board for the study contributed to its concept and design. Baxalta participated in the interpretation of the data and development of the manuscript.

\section{Disclosure}

Sophie le Doré, Nathalie Grinda, and Emmanuelle Ferré state that they have no interests that might be perceived as posing a conflict or bias. Valerie Roussel-Robert is an unpaid consultant for Shire (a Takeda company) and reports grants from APHP, during the conduct of the study. Birgit Frotscher has received grant/research support from CSL Behring, Shire (a Takeda company), Baxter, SOBI, Octapharma, Novo Nordisk, and Pfizer. Pierre Chamouni has received grant/research support from CSL Behring, Novo Nordisk, Shire (a Takeda company), and SOBI, and is a speaker for LFB. Sandrine Meunier has received grant/research support from Biogen/SOBI, CSL Behring, Novo Nordisk, Pfizer, Baxter, and Shire (a Takeda company); is a consultant for Novo Nordisk, Roche, and Shire (a Takeda company); and is a speaker bureau member for CSL Behring and Shire (a Takeda company). Sophie Bayart has received grant/research support from Shire (a Takeda company) and Sobi. Edita Dolimier was an employee of Baxalta France SAS (a Takeda company) at the time this study was performed. Francoise Truong-Berthoz is an employee of Baxalta GmbH (a Takeda company). Emmanuelle de Raucourt has received grant/research support from CSL Behring, and consulting and/or travel fees from Alexion, Bayer, CSL Behring, Novo Nordisk, Sobi, and Shire (a Takeda company). The authors report no other conflicts of interest in this work.

\section{References}

1. Lobet S, Hermans C, Lambert C. Optimal management of haemophilic arthropathy and hematomas. J Blood Med. 2014;5:207-218. doi:10. $2147 / \mathrm{jbm} . \mathrm{s} 50644$

2. Rodriguez-Merchan EC. Articular bleeding in hemophilia. Cardiovasc Hematol Disord Drug Targets. 2016;16(1):21-24. doi:10.2174/18715 29x16666160613114506

3. Rodriguez-Merchan EC. Musculoskeletal complications of hemophilia. HSS J. 2010;6(1):37-42. doi:10.1007/s11420-009-9140-9

4. Rodriguez-Merchan EC. Musculo-skeletal manifestations of haemophilia. Blood Rev. 2016;30(5):401-409. doi:10.1016/j.blre.2016. 04.008

5. Rodriguez-Merchan EC, De la Corte-rodriguez H, Jimenez-Yuste V. Joint aspiration of acute tense knee haemarthroses in adult haemophilia A patients. Thromb Res. 2013;132(6):778-779. doi:10.1016/j. thromres.2013.09.025

6. De la Corte-rodriguez H, Rodriguez-Merchan EC, Alvarez-Roman MT, Martin-Salces M, Romero-Garrido JA, Jimenez-Yuste V. Accelerating recovery from acute hemarthrosis in patients with hemophilia: the role of joint aspiration. Blood Coagul Fibrinolysis. 2019;30 (3):111-119. doi:10.1097/MBC.0000000000000803 
7. Rodriguez-Merchan EC. Management of musculoskeletal complications of hemophilia. Semin Thromb Hemost. 2003;29(1):87-96. doi:10.1055/s-2003-37942

8. Liddle AD, Rodriguez-Merchan EC. Evidence-based management of the knee in hemophilia. JBJS Rev. 2017;5(8):e12. doi:10.2106/JBJS. RVW.16.00100

9. De la Corte-rodriguez H, Rodriguez-Merchan EC. The role of physical medicine and rehabilitation in haemophiliac patients. Blood Coagul Fibrinolysis. 2013;24(1):1-9. doi:10.1097/MBC.0b013e32 $835 \mathrm{a} 72 \mathrm{f3}$

10. De la Corte-rodriguez H, Rodriguez-Merchan EC, Alvarez-Roman T, Martin-Salces M, Jimenez-Yuste V. 'Do not do' recommendations in hemophilia. Cardiovasc Hematol Disord Drug Targets. 2020;20 (3):168-174. doi:10.2174/1871529X20666200305111323

11. Rodriguez-Merchan EC, Hedner U, Heijnen L, et al. Prevention of haemophilic arthropathy during childhood. May common orthopaedic management be extrapolated from patients without inhibitors to patients with inhibitors? Haemophilia. 2008;14(Suppl 6):68-81. doi:10.1111/j.1365-2516.2008.01892.x

12. Rodriguez-Merchan EC, Jimenez-Yuste V, Aznar JA, et al. Joint protection in haemophilia. Haemophilia. 2011;17(Suppl 2):1-23. doi:10.1111/j.1365-2516.2011.02615.x

13. Rodriguez-Merchan EC. Prevention of the musculoskeletal complications of hemophilia. Adv Prev Med. 2012;2012:201271. doi:10.1155/ 2012/201271

14. Berntorp E, Astermark J, Björkman S, et al. Consensus perspectives on prophylactic therapy for haemophilia: summary statement. Haemophilia. 2003;9(Suppl 1):1-4. doi:10.1046/j.1365-2516.9.s1.17.x

15. Pérez-Robles T, Romero-Garrido JA, Rodriguez-Merchan EC, Herrero-Ambrosio A. Objective quantification of adherence to prophylaxis in haemophilia patients aged 12 to 25 years and its potential association with bleeding episodes. Thromb Res. 2016;143:22-27. doi:10.1016/j.thromres.2016.04.015

16. El Dakhakhny AM, Hesham MA, Hassan TH, El Awady S, Hanfy MM. Impact of health instructions on improving knowledge and practices of haemophilia A adolescents: a single centre experience. Haemophilia. 2014;20(4):e260-e266. doi:10.1111/hae.12431

17. D'Ivernois JF, Gagnayre R. Learning to Educate the Patient: Pedagogical Approach. 5th ed. [French]. Paris, France: Maloine; 2016.
18. Schrijvers LH, Beijlevelt-van der ZANDE M, Peters M, Schuurmans MJ, Fischer K. Learning intravenous infusion in haemophilia: experience from the Netherlands. Haemophilia. 2012;18 (4):516-520. doi:10.1111/j.1365-2516.2012.02752.x

19. Schrijvers LH, Schuurmans MJ, Fischer K. Promoting self-management and adherence during prophylaxis: evidence-based recommendations for haemophilia professionals. Haemophilia. 2016;22(4):499-506. doi:10.1111/hae.12904

20. Phadnis S, Kar A. The impact of a haemophilia education intervention on the knowledge and health related quality of life of parents of Indian children with haemophilia. Haemophilia. 2017;23(1):82-88. doi:10.1111/hae. 13070

21. Castaman G, Rocino A, Mazzucconi MG, Zanon E, Gagliano F, Molinari AC. Prophylaxis therapy in paediatric patients with haemophilia: a survey of clinical management trends in Italy. Blood Transfus. 2015;13(4):631-638. doi:10.2450/2015.0333-14

22. Berntorp E. Joint outcomes in patients with haemophilia: the importance of adherence to preventive regimens. Haemophilia. 2009;15 (6):1219-1227. doi:10.1111/j.1365-2516.2009.02077.x

23. Petrini P. Identifying and overcoming barriers to prophylaxis in the management of haemophilia. Haemophilia. 2007;13(Suppl 2):16-22. doi:10.1111/j.1365-2516.2007.01501.x

24. Geraghty S, Dunkley T, Harrington C, Lindvall K, Maahs J, Sek J. Practice patterns in haemophilia A therapy - global progress towards optimal care. Haemophilia. 2006;12(1):75-81. doi:10.1111/j.13652516.2006.01189.x

25. Petrini P, Seuser A. Haemophilia care in adolescents - compliance and lifestyle issues. Haemophilia. 2009;15(Suppl 1):15-19. doi:10.11 11/j.1365-2516.2008.01948.x

26. De la Corte-rodriguez H, Rodriguez-Merchan EC, Alvarez-Roman T, Martin-Salces M, Garcia-Barcenilla S, Jimenez-Yuste V. Health education and empowerment in adult patients with haemophilia. Expert Rev Hematol. 2019;12(11):989-995. doi:10.1080/17474086.2019. 1650640

27. Lambert C, Meité N, Sanogo I, Lobet S, Hermans C. Development and evaluation of appropriate, culturally adapted educational tools for Ivoirian patients with haemophilia, haemophilia carriers and their families. Haemophilia. 2019;25(5):838-844. doi:10.1111/hae.13818
Journal of Blood Medicine

\section{Publish your work in this journal}

The Journal of Blood Medicine is an international, peer-reviewed, open access, online journal publishing laboratory, experimental and clinical aspects of all aspect pertaining to blood based medicine including but not limited to: Transfusion Medicine; Blood collection, Donor issues, Transmittable diseases, and Blood banking logistics; Immunohematology; Artificial and alternative blood based therapeutics; Hematology; Biotechnology/nanotechnology of blood related medicine; Legal aspects of blood medicine; Historical perspectives. The manuscript management system is completely online and includes a very quick and fair peer-review system. Visit http://www.dovepress.com/testimonials.php to read real quotes from published authors. 